\title{
Real Multiplication Revisited
}

\section{Nikolaev IV*}

Department of Mathematical Sciences, The Fields Institute for Research in Mathematical Sciences, Toronto, ON, Canada

\begin{abstract}
It is proved that the Hilbert class field of a real quadratic field $Q(\sqrt{D})$ modulo a power $m$ of the conductor $f$ is generated by the Fourier coefficients of the Hecke eigenform for a congruence subgroup of level $f D$.
\end{abstract}

\section{Keywords: Class field; Real multiplication}

\section{Introduction}

The Kronecker's Jugendtraum is a conjecture that the maximal unramified abelian extension (The Hilbert class field) of any algebraic number field is generated by the special values of modular functions attached to an abelian variety. The conjecture is true for the rational field and imaginary quadratic fields with the modular functions being an exponent and the $j$-invariant, respectively. In the case of an arbitrary number field, a description of the abelian extensions is given by class field theory, but an explicit formula for the generators of these abelian extensions, in the sense sought by Kronecker, is unknown even for the real quadratic fields.

The problem was first studied by Hecke [1]. A description of abelian extensions of real quadratic number fields in terms of coordinates of points of finite order on abelian varieties associated with certain modular curves was obtained in studies of Shimura [2]. Stark formulated a number of conjectures on abelian extension of arbitrary number fields, which in the real quadratic case amount to specifying generators of these extensions using special values of Artin $L$-functions [3]. Based on an analogy with complex multiplication, Manin suggested to use the so-called "pseudo-lattices" $\mathbb{Z}+\mathbb{Z} \theta$ in $\mathbb{R}$ having non-trivial real multiplications to produce abelian extensions of real quadratic fields [4]. Similar to the case of complex multiplication, the endomorphism ring $\Re_{\mathfrak{f}}=\mathbb{Z}+\mathfrak{f} O_{\mathrm{E}}$ of pseudo-lattice $\mathbb{Z}+\mathbb{Z} \theta$ is an order in the real quadratic field $\mathfrak{k}=\mathbb{Q}(\theta)$, where $O_{\mathfrak{k}}$ is the ring of integers of $\mathfrak{k}$ and $\mathfrak{f}$ is the conductor of $\mathfrak{R}_{\mathfrak{f}}$; Manin calls these pseudo-lattices with real multiplication.

The aim of our note is a formula for generators of the Hilbert class field of real quadratic fields based on a modularity and a symmetry of complex and real multiplication. To give an idea, let

$$
\Gamma_{1}(N)=\left\{\left(\begin{array}{ll}
a & b \\
c & d
\end{array}\right) \in S L_{2}(\mathbb{Z}) \mid a \equiv d \equiv 1 \bmod N, c \equiv 0 \bmod N\right\}
$$

be a congruence subgroup of level $N \geq 1$ and $\mathbb{H}$ be the Lobachevsky half-plane; let $X_{1}(N):=\mathbb{H} / \Gamma_{1}(N)$ be the corresponding modular curve and $S_{2}\left(\Gamma_{1}(N)\right)$ the space of all cusp forms on $\Gamma_{1}(N)$ of weight 2. Let $\varepsilon_{C M}^{(-D, f)}$ be elliptic curve with complex multiplication by an order $\mathfrak{R}_{\mathrm{f}}=\mathbb{Z}+\mathfrak{f} O_{\mathrm{k}}$ in the field $\mathfrak{k}=\mathbb{Q}(\sqrt{-D})$ [5]. Denote by $\mathcal{K}^{a b}(k):=k\left(j\left(\mathcal{E}_{C M}^{(-D, f)}\right)\right)$ the Hilbert class field of $k$ modulo conductor $f \geq 1$ and let $N=f D$; let $\operatorname{Jac}\left(X_{1}(f D)\right)$ be the Jacobian of modular curve $X_{1}(f D)$. There exists an abelian subvariety $A_{\phi} \subset J a c\left(X_{1}(f D)\right)$, such that its points of finite order generate $\mathbb{K}^{a b}(k),[2,6,7]$, Section 8 . The $\mathbb{K}^{a b}(k)$ is a $C M$-field, i.e. a totally imaginary quadratic extension of the totally real field $\mathbb{K}_{\varphi}$ generated by the Fourier coefficients of the Hecke eigenform $\phi(z) \in S_{2}\left(\Gamma_{1}(f D)\right)$ [2]. In particular, there exists a holomorphic map $X_{1}^{0}(f D) \rightarrow \varepsilon_{C M}^{(-D, f)}$, where $X_{1}^{0}(f D)$ is a
Riemann surface such that $\operatorname{Jac}\left(X_{1}^{0}(f D)\right) \cong A_{\phi}$; we refer to the above as a modularity of complex multiplication.

Recall that (twisted homogeneous) coordinate ring of an elliptic curve $\varepsilon(\mathbb{C})$ is isomorphic to a Sklyanin algebra, [8]; the norm-closure of a self-adjoint representation of the Sklyanin algebra by the linear operators on a Hilbert space $\mathcal{H}$ is isomorphic to a noncommutative torus $\mathcal{A}_{\theta}$, [9] for the definition.

Whenever elliptic curve $\varepsilon(C) \cong \varepsilon_{C M}^{(-D, f)}$ has complex multiplication, the noncommutative torus $\mathcal{A}_{\theta}$ has real multiplication by an order $\mathfrak{R}_{\mathfrak{f}}=\mathbb{Z}+\mathfrak{f} O_{\mathfrak{k}}$ in the field $\mathfrak{k}=\mathbb{Q}(\sqrt{-D})$; moreover, it is known that $\mathfrak{f}=f^{m}$ for the minimal power $m$ satisfying an isomorphism:

$$
\mathrm{Cl}\left(\mathfrak{R}_{f^{m}}\right) \cong \mathrm{Cl}\left(R_{f}\right),
$$

where $C l\left(R_{f}\right)$ and $C l\left(\Re_{f}\right)$ are the ideal class groups of orders $R_{f}$ and $\Re_{f}$, respectively. We shall refer to (2) as a symmetry of complex and real multiplication. The noncommutative torus with real multiplication by $\mathfrak{R}_{\text {f }}$ will be denoted by $\mathcal{A}_{R M}^{(D, f)}$.

Remark 1: The isomorphism (2) can be calculated using the wellknown formula for the class number of a non-maximal order $\mathbb{Z}+f O_{K}$ of a quadratic field $K=\mathbb{Q}(\sqrt{D})$ :

$$
h_{\mathbb{Z}+f O_{K}}=\frac{h_{O_{K}} f}{e_{f}} \prod_{p \mid f}\left(1-\left(\frac{D}{p}\right) \frac{1}{p}\right),
$$

where $h_{O_{K}}$ is the class number of the maximal order $O_{K}, e_{f}$ is the index of the group of units of $\mathbb{Z}+f O_{K}$ in the group of units of $O_{K}, p$ is a prime number and $\frac{D}{p}$ is the Legendre symbol $[10,11]$.

The (twisted homogeneous) coordinate ring of the Riemann surface $X_{1}^{0}(f D)$ is an AF-algebra $\mathbb{A}_{\phi^{0}}$ linked to a holomorphic differential $\phi^{0}(z) d z$ on $X_{1}^{0}(f D)$, see Section 2.2, Definition 1 and Remark 5 for the details; the Grothendieck semigroup $K_{0}^{+}\left(\mathbb{A}_{0}\right)$ is a pseudo-lattice $\mathbb{Z}+\mathbb{Z} \theta_{1}+\ldots+\mathbb{Z} \theta_{n-1}$ in the number field $\mathbb{K}_{\varphi,}$, where $n$ equals the genus of $X_{1}^{0}(f D)$. Moreover, a holomorphic map $X_{1}^{0}(f D) \rightarrow \mathcal{E}_{C M}^{(-D, f)}$ induces the $C^{*}$-algebra homomorphism $\mathbb{A}_{0} \rightarrow \mathcal{A}_{R M}^{(D, f)}$ between the corresponding coordinate rings, so that the following diagram commutes:

${ }^{*}$ Corresponding authors: Nikolaev IV, Professor, Department of Mathematical Sciences, The Fields Institute for Research in Mathematical Sciences, 222 College Street, Toronto, Ontario, Canada, Tel: 416-348-9710; E-mail: igor.v.nikolaev@gmail.com

Received December 22, 2015; Accepted January 25, 2016; Published January 28, 2016

Citation: Nikolaev IV (2016) Real Multiplication Revisited. J Generalized Lie Theory Appl S2: 007. doi:10.4172/1736-4337.S2-007

Copyright: (c) 2016 Nikolaev IV. This is an open-access article distributed under the terms of the Creative Commons Attribution License, which permits unrestricted use, distribution, and reproduction in any medium, provided the original author and source are credited. 


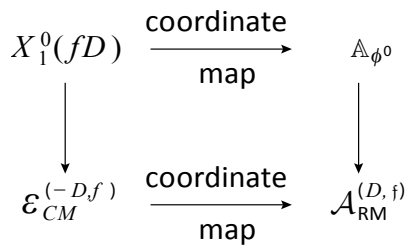

But $K_{0}^{+}\left(\mathcal{A}_{R M}^{(D, f)}\right)$ is a pseudo-lattice $\mathbb{Z}+\mathbb{Z} \theta$ in the field $\mathfrak{k}$, such that End $(\mathbb{Z}+\mathbb{Z} \theta) \cong \mathfrak{R}_{f}$; in other words, one can use the above diagram to control the arithmetic of the field $\mathcal{K}_{\varphi}$ by such of the real quadratic field $\mathfrak{k}$. Roughly speaking, this observation solves the Kronecker's Jugendtraum for the real quadratic fields; namely, the following is true.

Theorem 1. The Hilbert class field of a real quadratic field $\mathfrak{k}=\mathbb{Q}(\sqrt{D})$ modulo conductor $f^{m}$ is an extension of $\mathfrak{k}$ by the Fourier coefficients of the Hecke eigenform $\phi(z) \in S_{2}\left(\Gamma_{1}(f D)\right)$, where $m$ is the smallest positive integer satisfying isomorphism (2).

Remark 2. Theorem 1 can be used to compute concrete extensions. For instance, theorem 1 says that for the quadratic field $\mathbb{Q} \sqrt{15}$ its Hilbert class field is isomorphic to $\mathbb{Q} \sqrt{-1+\sqrt{15}}$ and for $\mathbb{Q} \sqrt{14}$ such a field modulo conductor $\mathfrak{f}=8$ is isomorphic to $\mathbb{Q}(\sqrt[4]{-27+8 \sqrt{14}})$ see section 4 for more examples.

The article is organized as follows. Section 2 covers basic facts on real multiplication and AF-algebras of the Hecke eigenforms. Theorem 1 is proved in Section 3. Section 4 contains numerical examples illustrating theorem 1 .

\section{Preliminaries}

The reader can find basics of the $C^{\star}$-algebras in studies of Murphy [12] and their $K$-theory in theory of Blackadar [13]. The noncommutative tori are covered in literature of Rieffel [9] and real multiplication in studies of Manin [4]. For main ideas of non-commutative algebraic geometry, see the survey by Stafford and van den Bergh [8]. The AFalgebras are reviewed in studies of Effros [14]. For a general theory of modular forms we refer to literature of Diamond and Shurman [15].

\section{Real multiplication}

The noncommutative torus $\mathcal{A}_{\theta}$ is a universal $C^{*}$-algebra generated by the unitary operators $u$ and $v$ acting on a Hilbert space $\mathcal{H}$ and satisfying the commutation relation $v u=e^{2 \pi i \theta} u v$, where $\theta$ is a real number. The $C^{*}$-algebra $\mathcal{A}_{\theta}$ is said to be stably isomorphic (Morita equivalent) to $\mathcal{A}_{\theta^{\prime}}$, whenever $\mathcal{A}_{\theta} \otimes \mathcal{K} \cong \mathcal{A}_{\theta^{\prime}} \otimes \mathcal{K}$, where $\mathcal{K}$ is the $C^{*}$ algebra of all compact operators on $\mathcal{H}$; the $\mathcal{A}_{\theta}$ is stably isomorphic to $\mathcal{A}_{\theta^{\prime}}$ if and only if

$$
\theta^{\prime}=\frac{a \theta+b}{c \theta+d} \text { for some matrix }\left(\begin{array}{ll}
a & b \\
c & d
\end{array}\right) \in S L_{2}(\mathbb{Z})
$$

The $K$-theory of $\mathcal{A}_{\theta}$ is two-periodic and $K_{0}\left(\mathcal{A}_{\theta}\right) \cong K_{1}\left(\mathcal{A}_{\theta}\right) \cong \mathbb{Z}^{2}$ so that the Grothendieck semigroup $K_{0}^{+}\left(\mathcal{A}_{\theta}\right)$ corresponds to positive reals of the pseudo-lattice $\mathbb{Z}+\mathbb{Z} \theta \subset \mathbb{R}$. The $\mathcal{A}_{\theta}$ is said to have real multiplication, if $\theta$ is a quadratic irrationality, i.e. irrational root of a quadratic polynomial in $\mathbb{Z}[x]$. The real multiplication says that the endomorphism ring of pseudo-lattice $\mathbb{Z}+\mathbb{Z} \theta$ exceeds the ring $\mathbb{Z}$ corresponding to multiplication by $m$ endomorphisms; similar to complex multiplication, it means that the endomorphism ring is isomorphic to an order $\mathfrak{R}_{\mathrm{f}}=\mathbb{Z}+\mathfrak{f} O_{\mathfrak{k}}$ of conductor $f \geq 1$ in the real quadratic field $\mathrm{k}=\mathbb{K}(\theta)$, hence the name. If $D>0$ is the discriminant of $\mathrm{k}$, then by $\mathcal{A}_{R M}^{(D, f)}$ we denote torus $\mathbb{A}_{\theta}$ with real multiplication by the order $\mathfrak{R}_{\mathrm{f}}$.
The Sklyanin algebra $S_{\alpha, \beta, \gamma}(\mathbb{C})$ is a free $C$-algebra on four generators and six relations:

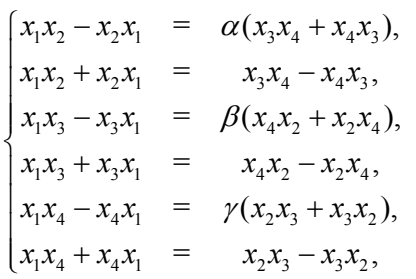

where $\alpha+\beta+\gamma+\alpha \beta \gamma=0$; such an algebra corresponds to a twisted homogeneouscoordinate ring of an elliptic curvein the complex projective space $\mathbb{C} P^{3}$ given by the intersection of two quadric surfaces of the form $\varepsilon_{\alpha, \beta, \gamma}(\mathbb{C})=\left\{(u, v, w, z) \in \mathbb{C} P^{3} \mid u^{2}+v^{2}+w^{2}+z^{2}=\frac{1-\alpha}{1+\beta} v^{2}+\frac{1+\alpha}{1-\gamma} w^{2}+z^{2}=0\right\}$.

Being such a ring means that the algebra $S_{\alpha, \beta, \gamma}$ satisfies an isomorphism

$$
\operatorname{Mod}\left({ }_{\alpha, \beta, \gamma}(\mathbb{C})\right) / \operatorname{Tors} \operatorname{Coh}\left({ }_{\alpha, \beta, \gamma}(\mathbb{C})\right),
$$

where Coh is the category of quasi-coherent sheaves on $\varepsilon_{\alpha, \beta, \gamma}(\mathbb{C})$, Mod the category of graded left modules over the graded ring $S_{\alpha, \beta, \gamma}(\mathbb{C})$ and Tors the full sub-category of Mod consisting of the torsion modules, [8].

If one sets $x_{1}=u, x_{2}=u^{*}, x_{3}=v, x_{4}=v^{*}$, then there exists a self-adjoint representation of the Sklyanin *-algebra $S_{\alpha, 1,-1}(\mathbb{C})$ by linear operators on a Hilbert space $\mathcal{H}$, such that its norm-closure is isomorphic to $\mathcal{A}_{\theta}$; namely, $\mathcal{A}_{\theta}^{0} \cong S_{\alpha, 1,-1}(\mathbb{C}) / I_{\mu}$ where $\mathcal{A}_{\theta}^{0}$ is a dense sub-algebra of $\mathcal{A}_{\theta}$ and $I_{\mu}$ is an ideal generated by the "scaled unit" relations $x_{1} x_{3}=x_{3} x_{4}=\frac{1}{\mu} e$, where $\mu>0$ is a constant. Thus the algebra $\mathcal{A}_{\theta}$ is a coordinate ring of elliptic curve $E(C)$, such that isomorphic elliptic curves correspond to the stably isomorphic (Morita equivalent) noncommutative tori; this fact explains the modular transformation law in (4). In particular, if $\varepsilon(\mathbb{C})$ has complex multiplication by an order $\Re_{\mathrm{f}}=\mathbb{Z}+\mathfrak{f} O_{\mathfrak{\varepsilon}}$ in a quadratic field $\mathfrak{k}=\mathbb{Q}(\sqrt{-D})$, then $\mathcal{A}_{\theta}$ has real multiplication by an order $\mathfrak{R}_{\mathrm{f}}=\mathbb{Z}+\mathfrak{f} O_{\mathfrak{k}}$ in the quadratic field $\mathfrak{k}=\mathbb{Q}(\sqrt{D})$, where $\mathrm{f}$ is the smallest integer satisfying an isomorphism $\mathrm{Cl}\left(\Re_{\mathrm{f}}\right) \cong \mathrm{Cl}\left(R_{f}\right)$, [16]; the isomorphism is a necessary and sufficient condition for $\mathcal{A}_{R M}^{(D, f)}$ to discern non-isomorphic elliptic curves $\varepsilon_{C M}^{(-D, f)}$ having the same endomorphism ring $R_{f}$. For the constraint $\mathfrak{f}=$ $f^{m}$, see remark 6 .

\section{AF-algebra of the Hecke eigenform}

An AF-algebra (Approximately Finite $C^{*}$-algebra) is defined to be the norm closure of an ascending sequence of finite dimensional $C^{*}$-algebras $M_{n}$, where $M_{n}$ is the $C^{\star}$-algebra of the $n \times n$ matrices with entries in $\mathbb{C}$. Here the index $n=\left(n_{1}, \ldots, n_{k}\right)$ represents the semi-simple matrix algebra $M_{n}=M_{n_{1}} \oplus \ldots \oplus M_{n_{b}}$. The ascending sequence mentioned above can be written as $M_{1} \stackrel{\varphi_{1}}{\longrightarrow} M_{2} \stackrel{\varphi_{2}}{\longrightarrow} \ldots$, where $M_{i}$ are the finite dimensional $C^{\star}$-algebras and $\phi_{i}$ the homomorphisms between such algebras. The homomorphisms $\phi_{i}$ can be arranged into a graph as follows. Let $M_{i}=M_{i_{1}} \oplus \ldots \oplus M_{i_{k}}$ and $M_{i^{\prime}}=M_{i_{1}} \oplus \ldots \oplus M_{i_{k^{\prime}}}$ be the semi-simple $C^{*}$ algebras and $\varphi_{i}: M_{i} \rightarrow M_{i^{\prime}}$ the homomorphism. One has two sets of vertices $V_{i_{1}}, \ldots, V_{i_{k}}$ and $V_{i_{1}}, \ldots, V_{i_{k^{\prime}}}$ joined by $b_{r s}$ edges whenever the summand $M_{i_{r}}$ contains $b_{r s}$ copies of the summand $M_{i,}$ under the embedding $\phi_{i}$. As varies, one obtains an infinite graph called the Bratteli diagram of the AFalgebra. The matrix $B=\left(b_{r s}\right)$ is known as a partial multiplicity matrix; an infinite sequence of $B_{i}$ defines a unique AF-algebra. An AF-algebra is called stationary if $B_{i}=$ Const $=B$, [14], when two non-similar matrices $B$ and $B$ have the same characteristic polynomial, the corresponding stationary AFalgebras will be called companion AF-algebras. 
Let $N \geq 1$ be a natural number and consider a (finite index) subgroup of the modular group given by the formula:

$$
\Gamma_{1}(N)=\left\{\left(\begin{array}{ll}
a & b \\
c & d
\end{array}\right) \in S L_{2}(\mathbb{Z}) \mid a \equiv d \equiv 1 \bmod N, c \equiv 0 \bmod N\right\} .
$$

Let $\mathbb{H}=\{z=x+i y \in \mathbb{C} \mid y>0\}$ be the upper half-plane and let $\Gamma_{1}(N)$ act on $\mathbb{H}$ by the linear fractional transformations; consider an orbifold $\mathbb{H} / \Gamma_{1}(N)$. To compactify the orbifold at the cusps, one adds a boundary to $\mathbb{H}$, so that $\mathbb{H}^{*}=\mathbb{H} \cup \mathbb{Q} \cup\{\infty\}$ and the compact Riemann surface $X_{1}(N)=\mathbb{H}^{*} / \Gamma_{1}(N)$ is called a modular curve. The meromorphic functions $(z)$ on $\mathbb{H}$ that vanish at the cusps and such that

$$
\phi\left(\frac{a z+b}{c z+d}\right)=(c z+d)^{2} \phi(z), \quad \forall\left(\begin{array}{ll}
a & b \\
c & d
\end{array}\right) \in \Gamma_{0}(N),
$$

are called cusp forms of weight two; the (complex linear) space of such forms will be denoted by $S_{2}\left(\Gamma_{1}(N)\right)$. The formula $\phi(z) \mapsto \omega=\phi(z) d z$ defines an isomorphism $S_{2}\left(\Gamma_{1}(N)\right) \cong \Omega_{h o l}\left(X_{1}(N)\right)$, where $\Omega_{h o l}\left(X_{1}(N)\right)$ is the space of all holomorphic differentials on the Riemann surface $X_{1}(N)$. Note that $\operatorname{dim}_{\mathbb{C}}\left(S_{2}\left(\Gamma_{1}(N)\right)=\operatorname{dim}_{\mathbb{C}}\left(\Omega_{h o l}\left(X_{1}(N)\right)=g\right.\right.$, where $g=g(N)$ is the genus of the surface $X_{1}(N)$. A Hecke operator, $T_{n}$, acts on $S_{2}\left(\Gamma_{1}(N)\right)$ by the formula $T_{n} \phi=\sum_{m \in Z^{\prime}} \gamma(m) q^{m}$, where $\gamma(m)=\sum_{a \mid \mathbf{G}} \mathbf{G C D} \mathbf{D}_{(m, n)} a{ }^{m}{ }_{m / a^{2}}$ and $\phi(z)=\sum_{m \in Z} c(m) q^{m}$ is the Fourier series of the cusp form $\phi$ at $q=e^{2 \pi i z}$. Further, $T_{n}$ is a self-adjoint linear operator on the vector space $S_{2}\left(\Gamma_{1}(N)\right)$ endowed with the Petersson inner product; the algebra $\mathbb{T}_{N}:=\mathbb{Z}\left[T_{1}, T_{2}, \ldots\right]$ is a commutative algebra. Any cusp form $\phi \in S_{2}\left(\Gamma_{1}(N)\right)$ that is an eigenvector for one (and hence all) of $T_{n}$, is referred to as a Hecke eigenform. The Fourier coefficients $c(m)$ of $\phi$ are algebraic integers, and we denote by $K_{\phi}=Q(c(m))$ an extension of the field $Q$ by the Fourier coefficients of $\phi$. Then $\mathcal{K}_{\varphi}$ is a real algebraic number field of degree $1 \leq \operatorname{deg}\left(\mathcal{K}_{\phi} \mid \mathbb{Q}\right) \leq g$, where $g$ is the genus of the surface $X_{1}(N)$ [5], Proposition 6.6.4. Any embedding $\sigma: \mathcal{K}_{\phi} \rightarrow \mathbb{C}$ conjugates $\phi$ by acting on its coefficients; we write the corresponding Hecke eigenform $\phi^{\sigma}(z):=\sum_{m \in Z} \sigma(c(m)) q^{m}$ and call $\phi^{\sigma}$ a conjugate of the Hecke eigenform $\phi$.

Let $\omega=\phi(z) d z \in \Omega_{h o l}(X)$ be a holomorphic differential on a Riemann surface $X$. We shall denote by $\mathrm{R}(w)$ a closed form on $X$ (the real part of $w$ ) and consider its periods $\lambda_{i}=\int_{\gamma_{i}} \mathfrak{R}(\omega)$ against a basis $\gamma_{i}$ in the (relative) homology group $H_{1}(X, Z(\Re(\omega)) ; \mathbb{Z})$, where $Z(\Re(\omega))$ is the set of zeros of the form $\mathrm{R}(w)$. Assume $\lambda_{i}>0$ and consider the vector $\theta=\left(\theta_{1}, \ldots, \theta_{n-1}\right)$ with $\theta_{i}=\lambda_{i+1} / \lambda_{1}$. The Jacobi-Perron continued fraction of $\theta$ is given by the formula:

$$
\left(\begin{array}{l}
1 \\
\theta
\end{array}\right)=\lim _{i \rightarrow \infty}\left(\begin{array}{ll}
0 & 1 \\
I & b_{1}
\end{array}\right) \ldots\left(\begin{array}{ll}
0 & 1 \\
I & b_{i}
\end{array}\right)\left(\begin{array}{l}
0 \\
\mathbb{I}
\end{array}\right)=\lim _{i \rightarrow \infty} B_{i}\left(\begin{array}{l}
0 \\
\mathbb{I}
\end{array}\right),
$$

where $b_{i}=\left(b_{1}^{(i)}, \ldots, b_{n-1}^{(i)}\right)^{T}$ is a vector of non-negative integers, $I$ is the unit matrix and $\mathbb{I}=(0, \ldots, 0,1)^{T}[17]$. By $\mathbb{A}_{\phi}$ we shall understand the AFalgebra given the Bratteli diagram with partial multiplicity matrices $B_{i}$. If $\phi(z) \in S_{2}\left(\Gamma_{1}(N)\right)$ is a Hecke eigenform, then the corresponding AFalgebra $\mathbb{A}_{\phi}$ is stationary with the partial multiplicity matrices $B_{i}=$ Const $=B$; moreover, each conjugate eigenform $\phi^{\sigma}$ defines a companion AFalgebra $A_{\sigma^{\sigma}}$. It is known that $K_{0}^{+}\left(\mathbb{A}_{\phi}\right) \cong \mathbb{Z}+\mathbb{Z} \theta_{1}+\ldots+\mathbb{Z} \theta_{n-1} \subset \mathcal{K}_{\phi}$, where $\mathcal{K}_{\phi}$ is an algebraic number field generated by the Fourier coefficients of $\phi,[18]$.

\section{Proof of Theorem 1}

Definition 1. Let $A_{\phi} \subset J a c\left(X_{1}(f D)\right)$ be an abelian variety associated to the Hecke eigenform $\phi(z) \in S_{2}\left(\Gamma_{1}(f D)\right)$ [15], Definition 6.6.3. By $X_{1}^{0}(f D)$ we shall understand the Riemann surface of genus $g$, such that

$$
\operatorname{Jac}\left(X_{1}^{0}(f D)\right) \cong A_{\phi} .
$$

By $\phi^{0}(z) d z \in \Omega_{\text {hol }}\left(X_{1}^{0}(f D)\right)$ we denote the image of the Hecke eigenform $\phi(z) d z \in \Omega_{h o l}\left(X_{1}(f D)\right)$ under the holomorphic map $X_{1}(f D) \rightarrow X_{1}^{0}(f D)$.

Remark 3. The surface $X_{1}^{0}(f D)$ is correctly dened. Indeed, since the abelian variety $A_{\varphi}$ is the product of $g$ copies of an elliptic curve with the complex multiplication, there exists a holomorphic map from $A$ to the elliptic curve. For a Riemann surface $X$ of genus $g$ covering the elliptic curve $\varepsilon_{C M}$ by a holomorphic map (such a surface and a map always exist), one gets a period map $X \rightarrow A_{\varphi}$ by closing the arrows of a commutative diagram $A_{\varphi} \rightarrow \varepsilon_{C M} \leftarrow X$. It is easy to see, that the Jacobian of $X$ coincides with $A_{\varphi}$ and we set $X_{1}^{0}(f D):=X$.

Lemma 1. $g\left(X_{1}^{0}(f D)\right)=\operatorname{deg}\left(\mathcal{K}^{a b}(k) \mid k\right)$.

Proof. By definition, abelian variety $A_{\varphi}$ is the quotient of $\mathbb{C}^{n}$ by a lattice of periods of the Hecke eigenform $\phi(z) \in S_{2}\left(\Gamma_{1}(f D)\right)$ and all its conjugates $\phi^{\sigma}(z)$ on the Riemann surface $X_{1}(f D)$. These periods are complex algebraic numbers generating the Hilbert class field $K^{a b}(k)$ over imaginary quadratic field $k=Q(\sqrt{-D})$ modulo conductor $f,[2,6,7]$, Section 8 . The number of linearly independent periods is equal to the total number of the conjugate eigenforms $\phi^{\sigma}(z)$, i.e. $|\sigma|=n=\operatorname{dim}_{\mathbb{C}}\left(A_{\phi}\right)$. Since real dimension $\operatorname{dim}_{\mathbb{R}}\left(A_{\phi}\right)=2 n$, we conclude that $\operatorname{deg}\left(\mathcal{K}^{a b}(k) \mid \mathbb{Q}\right)=2 n$ and, therefore, $\operatorname{deg}\left(\mathcal{K}^{a b}(k) \mid k\right)=n$. But $\operatorname{dim}_{\mathbb{C}}\left(A_{\phi}\right)=g\left(X_{1}^{0}(f D)\right)$ and one gets $g\left(X_{1}^{0}(f D)\right)=\operatorname{deg}\left(\mathcal{K}^{a b}(k) \mid k\right)$. Lemma 1 follows.

Corollary 1. $g\left(X_{1}^{0}(f D)\right)=\left|C l\left(R_{f}\right)\right|$.

Proof. Because $\mathcal{K}^{a b}(k)$ is the Hilbert class field over $k$ modulo conductor $f$, we must have

$$
\operatorname{Gal}\left(\mathcal{K}^{a b}(k) \mid k\right) \cong C l\left(R_{f}\right),
$$

where $\mathrm{Gal}$ is the Galois group of the extension $\mathcal{K}^{a b}(k) \mid k$ and $\mathrm{Cl}\left(R_{f}\right)$ is the class group of ring $R_{\rho}$ [5]. But $\left|\operatorname{Gal}\left(\mathcal{K}^{a b}(k) \mid k\right)\right|=\operatorname{deg}\left(\mathcal{K}^{a b}(k) \mid k\right)$ and by lemma 1 we have $\operatorname{deg}\left(\mathcal{K}^{a b}(k) \mid k\right)=g\left(X_{1}^{0}(f D)\right)$. In view of this and isomorphism (11), one gets $\left|C l\left(R_{f}\right)\right|=\left|\operatorname{Gal}\left(\mathcal{K}^{a b} \mid k\right)\right|=g\left(X_{1}^{0}(f D)\right)$. Corollary 1 follows.

Lemma 2. $g\left(X_{1}^{0}(f D)\right)=\operatorname{deg}\left(\mathcal{K}_{\phi} \mid Q\right)$.

Proof. It is known that $\operatorname{dim}_{\mathbb{C}}\left(A_{\phi}\right)=\operatorname{deg}\left(\mathcal{K}_{\phi} \mid \mathbb{Q}\right)$ [15], Proposition 6.6.4. But abelian variety $A_{\phi} \cong \operatorname{Jac}\left(X_{1}^{0}(f D)\right)$ and, therefore, $\operatorname{dim}_{\mathbb{C}}\left(A_{\phi}\right)=\operatorname{dim}_{\mathbb{C}}\left(\operatorname{Jac}\left(X_{1}^{0}(f D)\right)\right)=g\left(X_{1}^{0}(f D)\right)$, hence the lemma.

Corollary 2. $\operatorname{deg}\left(\mathcal{K}_{\phi} \mid \mathbb{Q}\right)=\left|C l\left(\Re_{f}\right)\right|$.

Proof. From lemma 2 and corollary 1 one gets $\operatorname{deg}\left(\mathcal{K}_{\phi} \mid \mathbb{Q}\right)=\left|C l\left(R_{f}\right)\right|$. In view of this and equality (2), one gets the conclusion of corollary 2.

Lemma 3. (Basic lemma) $\operatorname{Gal}\left(\mathcal{K}_{\phi} \mid \mathbb{Q}\right) \cong C l\left(\Re_{f}\right)$.

Proof. Let us outline the proof. In view of lemma 2 and corollaries 1-2, we denote by $h$ the single integer $g\left(X_{1}^{0}(f D)\right)=\left|C l\left(R_{f}\right)\right|=\left|C l\left(\mathfrak{R}_{f}\right)\right|=\operatorname{deg}\left(\mathcal{K}_{\phi} \mid \mathbb{Q}\right)$. Since $\operatorname{deg}\left(\mathcal{K}_{\phi} \mid \mathbb{Q}\right)=h$, there exist $\left\{\phi_{1}, \ldots, \phi_{h}\right\}$ conjugate Hecke eigenforms $\phi_{i}(z) \in S_{2}\left(\Gamma_{1}(f D)\right)$ [15], Theorem 6.5.4; thus one gets $h$ holomorphic forms $\left\{\phi_{1}^{0}, \ldots, \phi_{h}^{0}\right\}$ on the Riemann surface $X_{1}^{0}(f D)$. Let $\left\{A_{\phi^{0}}, \ldots, A_{\phi^{0}}\right\}$ be the corresponding stationary AF-algebras; the $A_{0}$ are companion AFalgebras, see Section 1.2. Recall that the characteristic polynomial for the partial multiplicity matrices $B_{0^{0}}$ of companion AF-algebras $A_{0^{0}}$ is the same; it is a minimal polynomial of degree $h$ and let $\left\{\lambda_{1}, \ldots, \lambda_{h}^{\phi_{i}}\right\}$ be the roots of such a polynomial, compare with studies of Effros [14], Corollary 6.3. Since $\operatorname{det}\left(B_{\phi_{i}^{0}}\right)=1$, the numbers $\lambda_{i}$ are algebraic units of the field $\mathcal{K}_{\varphi}$. Moreover, $\lambda_{i}{ }_{i}$ are algebraically conjugate and can be taken for generators of the extension $\mathcal{K}_{\phi} \mid \mathbb{Q}$; since $\operatorname{deg}\left(\mathcal{K}_{\phi} \mid \mathbb{Q}\right)=h=\left|C l\left(\Re_{f}\right)\right|$ there exists a natural action of group $\mathrm{Cl}\left(\mathfrak{R}_{\mathrm{f}}\right)$ on these generators. The 
action extends to automorphisms of the entire field $\mathcal{K}_{\varphi}$ preserving $\mathbb{Q}$; thus one gets the Galois group of extension $\mathcal{K}_{\phi} \mid \mathbb{Q}$ and an isomorphism $\operatorname{Gal}\left(\mathcal{K}_{\phi} \mid \mathbb{Q}\right) \cong C l\left(\Re_{\mathrm{f}}\right)$. Let us pass to a step-by-step argument.

(i) Let $h:=g\left(X_{1}^{0}(f D)\right)=\left|C l\left(R_{f}\right)\right|=\left|C l\left(\Re_{f}\right)\right|$ and let $\phi(z) \in S_{2}\left(\Gamma_{1}(f D)\right)$ be the Hecke eigenform. It is known that there exists $\left\{\phi_{1}, \ldots, \phi_{h}\right\}$ conjugate Hecke eigenforms, so that $\phi(z)$ is one of them [15], Theorem 6.5.4. Let $\left\{\phi_{1}^{0}, \ldots, \phi_{h}^{0}\right\}$ be the corresponding forms on the Riemann surface $X_{1}^{0}(f D)$.

Remark 4. The forms $\left\{\phi_{1}^{0}, \ldots, \phi_{h}^{0}\right\}$ can be taken for a basis in the space $\Omega_{h o l}\left(X_{1}^{0}(f D)\right)$; we leave it to the reader to verify, that abelian variety $A_{\varphi}$ is isomorphic to the quotient of $\mathbb{C}^{h}$ by the lattice of periods of holomorphic differentials $\phi_{i}^{0}(z) d z$ on $X_{1}^{0}(f D)$.

(ii) Let $\mathbb{A}_{\phi_{i}^{0}}$ be the AF-algebra corresponding to holomorphic differential $\phi_{i}^{0}(z) d z$ on $X_{1}^{0}(f D)$, see Section 2.2; the set $\left\{\mathbb{A}_{\phi_{1}^{0}}, \ldots, \mathbb{A}_{\phi_{1}^{0}}\right\}$ consists of the companion AF-algebras. It is known that each $\mathbb{A}_{\phi_{0}^{0}}$ is a stationary AF-algebra, i.e. its partial multiplicity matrix is a constant; we shall denote such a matrix by $B_{\phi_{i}^{0}}$.

(iii) By definition, the matrices $B_{\phi^{0}}$ of companion $A F$-algebras $\mathbb{A}_{\phi_{i}}$ have the same characteristic polynomial $p(x) \in \mathbb{Z}[x]$; the matrices $B_{\phi_{0}^{0}}$ itself are not pairwise similar and, therefore, the AF-algebras $\mathbb{A}_{0}$ are not pairwise isomorphic. The total number $h$ of such matrices is equal to the class number of the endomorphism ring of pseudo-lattice $K_{0}^{+}\left(\mathbb{A}_{\phi^{0}}\right) \cong \mathbb{Z}+\mathbb{Z} \theta_{1}^{i}+\ldots+\mathbb{Z} \theta_{h-1}^{i} \subset \mathcal{K}_{\phi},[14]$.

Remark 5. Notice that there are $\left\{X_{1}, \ldots, X_{h}\right\}$ pairwise non-isomorphic Riemann surfaces $X:=X_{1}^{0}(f D)$ endowed with a holomorphic map $X_{i} \rightarrow$ $\varepsilon_{i}$ where, $\left\{\varepsilon_{1}, \ldots,{ }_{h}\right\}$ are pairwise non-isomorphic elliptic curves $\varepsilon_{C M}^{(-D, f)}$ corresponding to elements of the group $\mathrm{Cl}\left(R_{f}\right)$. Thus the companion AF-algebras $\left\{\mathbb{A}_{\phi^{0}}, \ldots, \mathbb{A}_{\phi^{0}}\right\}$ can be viewed as coordinate rings of $\left\{X_{1}\right.$ ,..., $\left.X_{h}\right\}$ the latter means that $\mathbb{A}_{\phi_{i}^{0}}$ discern non-isomorphic Riemann surfaces and $K_{0}^{+}\left(\mathbb{A}_{\phi^{0}}\right) \cong \mathbb{Z}+\mathbb{Z} \theta_{1}^{i}+\ldots+\mathbb{Z} \theta_{h-1}^{i} \subset \mathcal{K}_{\phi}$ represents the moduli space of $X_{1}^{0}(f D)$.

(iv) The polynomial $p(x)$ is minimal and splits in the totally real field $\mathcal{K}_{\varphi}$. Indeed, matrices ${ }_{2} \mathrm{~S}_{\mathrm{T}}(N)$ generate the Hecke algebra $\mathbb{T}_{\mathrm{N}}$ on $S_{2}\left(\Gamma_{1}(N)\right)$; thus each $B_{\phi_{1}^{0}}$ is self-adjoint and, therefore, all eigenvalues are real of multiplicity one; since $B_{\phi_{i}^{0}}$ is integer, all roots of characteristic polynomial $p(x)$ of $B_{\phi_{i}^{0}}$ belong to the field $\mathcal{K}_{\varphi}$.

(v) Let $p(x)=\left(x-\lambda_{1}\right) \ldots\left(x-\lambda_{h}\right)$. It is easy to see that $\lambda_{i}$ are algebraic units of the field $\mathcal{K}_{\varphi}$ because $\operatorname{det}\left(B_{\phi_{i}^{0}}\right)=1 ;$ note that numbers $\left\{\lambda_{1}, \ldots, \lambda_{h}\right\}$ are algebraically conjugate. Since $\operatorname{deg}\left(\mathcal{K}_{\phi} \mid \mathbb{Q}\right)=h$, the numbers $\lambda_{i}$ can be taken for generators of the field $\mathcal{K}_{\varphi}$, i.e. $\mathcal{K}_{\phi}=\mathbb{Q}\left(\lambda_{1}, \ldots, \lambda_{h}\right)$.

(vi) Finally, let us establish an explicit formula for the isomorphism

$$
\mathrm{Cl}\left(\mathfrak{R}_{\mathrm{f}}\right) \rightarrow \operatorname{Gal}\left(\mathcal{K}_{\phi} \mid \mathbb{Q}\right)
$$

Since $\operatorname{Gal}\left(\mathcal{K}_{\phi} \mid \mathbb{Q}\right)$ is an automorphism group of the field $\mathcal{K}$ preserving $\mathbb{Q}$, it will suffice to define the action of an element $a \in C l\left(\mathfrak{R}_{\mathrm{f}}\right)^{\varphi}$ on the generators $\lambda_{i}$ of $\mathcal{K}_{\varphi}$. Let $\left\{a_{1}, \ldots, a_{h}\right\}$ be the set of all elements of the group $\mathrm{Cl}\left(\mathfrak{R}_{\mathrm{f}}\right)$. For an element $a \in C l\left(\Re_{\mathrm{f}}\right)$ define an index function $\alpha$ by the formula $a_{i} a=a_{\alpha(i)}$. Then the action $*$ of an element $a \in C l\left(\Re_{\mathrm{f}}\right)$ on the generators $\lambda_{i}$ of the field $\mathcal{K}_{\varphi}$ is given by the formula:

$$
a * \lambda_{i}:=\lambda_{a(i)}, \quad \forall a \in C l\left(\Re_{\mathrm{f}}\right) .
$$

It is easy to verify that formula (13) gives an isomorphism $C l\left(\Re_{f}\right) \rightarrow \operatorname{Gal}\left(\mathcal{K}_{\phi} \mid \mathbb{Q}\right)$, which is independ of the choice of $\left\{a_{i}\right\}$ and $\left\{\lambda_{i}\right\}$. This argument completes the proof of lemma 3.
Remark 6. The class field theory says that $\mathfrak{f}=f^{m}$, i.e. the extensions of elds $k$ and $\mathfrak{k}$ must ramify over the same set of prime ideals. Indeed, consider the commutative diagram below, where $I_{f}$ and $I_{\mathrm{f}}$ are groups of all ideals of $k$ and $\mathfrak{k}$, which are relatively prime to the principal ideals $(f)$ and $(\mathfrak{f})$, respectively. Since $\operatorname{Gal}\left(\mathcal{K}^{a b}(k) \mid \mathbb{Q}\right) \cong \operatorname{Gal}\left(\mathcal{K}_{\phi} \mid \mathbb{Q}\right)$ one gets an isomorphism $I_{f} \cong I_{\mathrm{f}}$, i.e. $\mathfrak{f}=f^{m}$ for some positive integer $m$.

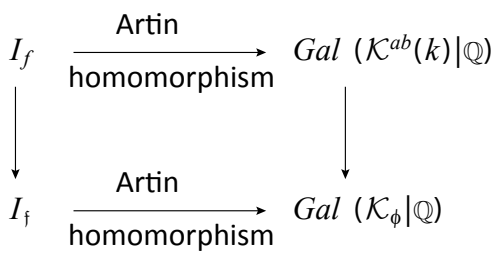

Corollary 3. The Hilbert class field of real quadratic field $\mathfrak{k}=Q(\sqrt{D})$ modulo conductor $\mathfrak{f} \geq 1$ is isomorphic to the field $\mathfrak{k}\left(\mathcal{K}_{\phi}\right)$ generated by the Fourier coefficients of the Hecke eigenform $\phi(z) \in S_{2}\left(\Gamma_{1}(f D)\right)$.

Proof. As in the classical case of imaginary quadratic fields, notice that $\operatorname{deg}\left(\mathcal{K}_{\phi} \mid \mathbb{Q}\right)=\operatorname{deg}\left(\mathfrak{k}\left(\mathcal{K}_{\phi}\right) \mid \mathfrak{k}\right)=C l\left(\Re_{\mathrm{f}}\right)$; therefore corollary 3 is an implication of lemma 3 and isomorphism $\operatorname{Gal}\left(\mathcal{K}_{\phi} \mid \mathbb{Q}\right)=\operatorname{Gal}\left(\mathfrak{k}\left(\mathcal{K}_{\phi}\right) \mid \mathfrak{k}\right)=\mathrm{Cl}\left(\mathfrak{R}_{\mathfrak{f}}\right)$.

Theorem 1 follows from corollary 3.

\section{Examples}

Along with the method of Stark's units [19], theorem 1 can be used in the computational number theory. For the sake of clarity, we shall consider the simplest examples; the rest can be found in Table 1.

Example 1. Let $D=15$. The class number of quadratic field $k=\mathbb{Q}(\sqrt{-15})$ is known to be 2 ; such a number for quadratic field $\mathfrak{k}=\mathbb{Q}(\sqrt{15})$ is also equal to 2 . Thus

$$
C l\left(\Re_{f=1}\right) \cong C l\left(R_{f=1}\right) \cong \mathbb{Z} / 2 \mathbb{Z},
$$

and isomorphism (2) is trivially satised for each power $m$, i.e. one obtains an unramied extension. By theorem 1, the Hilbert class field of $\mathrm{k}$ is generated by the Fourier coefficients of the Hecke eigenform $\phi(z) \in S_{2}\left(\Gamma_{1}(15)\right)$. Using the computer programme $S A G E$ created by William A. Stein, one finds an irreducible factor $p(x)=x^{2}-4 x+5$ of the characteristic polynomial of the Hecke operator $T_{p=2}$ acting on the space $S_{2}\left(\Gamma_{1}(15)\right)$. Therefore, the Fourier coefficient $c(2)$ coincides with a root of equation $p(x)=0$; in other words, we arrive at an extension of $\mathrm{k}$ by the polynomial $p(x)$. The generator $x$ of the field $\mathcal{K}_{\varphi}=\mathbb{Q}(c(2))$ is a root of the bi-quadratic equation $\left[(\mathrm{x} 2)^{2}+1\right]^{2}-15=0$; it is easy to see that $x=2+\sqrt{-1+\sqrt{15}}$. One concludes, that the field $\mathcal{K}_{\phi} \cong \mathbb{Q} \sqrt{-1+\sqrt{15}}$. is the Hilbert class field of quadratic field $\mathfrak{k}=\mathbb{Q} \sqrt{15}$.

Example 2. Let $D=14$. It is known, that for the quadratic field $k=\mathbb{Q}(\sqrt{-14})$ we have $\left|C l\left(R_{f=1}\right)\right|=4$, while for the quadratic field $C l\left(\Re_{f=1}\right)=1$. it holds $C l\left(\Re_{\mathrm{f}=1}\right)=1$. However, for the ramified extensions one obtains the following isomorphism:

$$
C l\left(\Re_{\mathrm{f}=2^{3}}\right) \cong C l\left(R_{f=2}\right) \cong \mathbb{Z} / 4 \mathbb{Z},
$$

where $m=3$ is the smallest integer satisfying formula (2). By theorem 1 , the Hilbert class field of $\mathfrak{k}$ modulo $\mathfrak{f}=8$ is generated by the Fourier coefficients of the Hecke eigenform $\varphi(z) \in S_{2}\left(\Gamma_{1}(2 \times 4)\right)$. Using the $S A G E$, one finds that the characteristic polynomial of the Hecke operator $T_{p=3}$ on $S_{2}\left(\Gamma_{1}(2 \times 4)\right)$ has an irreducible factor $p(x)=x^{4}+3 x^{2}+$ 9. Thus the Fourier coecient $c(3)$ is a root of the polynomial $p(x)$ and one gets an extension of $\mathfrak{k}$ by the polynomial $p(x)$. In other words, generator $x$ of the field $\mathcal{K}_{\varphi}=\mathbb{Q}(c(3))$ is a root of the polynomial equation $\left(x^{4}+\right.$ 


\begin{tabular}{|c|c|c|c|c|}
\hline$D$ & $f$ & $C l\left(R_{f}\right)$ & $f$ & $\begin{array}{l}\text { Hilbert class field of } \mathbb{Q}(\sqrt{D}) \\
\text { modulo conductor } f\end{array}$ \\
\hline 2 & 1 & trivial & 1 & $\mathbb{Q}(\sqrt{2})$ \\
\hline 3 & 1 & trivial & 1 & $\mathbb{Q}(\sqrt{3})$ \\
\hline 7 & 1 & trivial & 1 & $\mathbb{Q}(\sqrt{7})$ \\
\hline 11 & 1 & trivial & 1 & $\mathbb{Q}(\sqrt{11})$ \\
\hline 14 & 2 & $\mathbb{Z} / 4 \mathbb{Z}$ & 8 & $\mathbb{Q}(\sqrt[4]{-27+8 \sqrt{14}})$ \\
\hline 15 & 1 & $\mathbb{Z} / 2 \mathbb{Z}$ & 1 & $\mathbb{Q}(\sqrt{-1+\sqrt{15}})$ \\
\hline 19 & 1 & trivial & 1 & $\mathbb{Q}(\sqrt{19})$ \\
\hline 21 & 2 & $\mathbb{Z} / 4 \mathbb{Z}$ & 8 & $\mathbb{Q}(\sqrt[4]{-3+2 \sqrt{21}})$ \\
\hline 35 & 1 & $\mathbb{Z} / 2 \mathbb{Z}$ & 1 & $\mathbb{Q}(\sqrt{17+\sqrt{35}})$ \\
\hline 43 & 1 & trivial & 1 & $\mathbb{Q}(\sqrt{43})$ \\
\hline 51 & 1 & $\mathbb{Z} / 2 \mathbb{Z}$ & 1 & $\mathbb{Q}(\sqrt{17+\sqrt{51}})$ \\
\hline 58 & 1 & $\mathbb{Z} / 2 \mathbb{Z}$ & 1 & $\mathbb{Q}(\sqrt{-1+\sqrt{58}})$ \\
\hline 67 & 1 & trivial & 1 & $\mathbb{Q}(\sqrt{67})$ \\
\hline 82 & 1 & $x^{2}-2 x^{2}+2+x-5+2+16$ & 1 & $x^{4}-2 x^{3}+4 x^{2}-8 x+16$ \\
\hline 91 & 1 & $\mathbb{Z} / 2 \mathbb{Z}$ & 1 & $\mathbb{Q}(\sqrt{-3+\sqrt{91}})$ \\
\hline
\end{tabular}

Table 1: Square-free discriminants $2 \leq \mathrm{D} \leq 101$.

$\left.3 x^{2}+9\right)^{2}-4 \times 14=0$. The bi-quadratic equation $x^{4}+3 x^{2}+9-2 \sqrt{14}=0$ has discriminant $-27+8 \sqrt{14}$ and one finds a generator of $\mathcal{K}_{\varphi}$ to be $\sqrt[4]{-27+8 \sqrt{14}}$. Thus the field $\mathbb{Q} \sqrt[4]{-27+8 \sqrt{14}}$ is the Hilbert class over $\mathbb{Q}(\sqrt{14})$ modulo conductor $\mathfrak{f}=8$. Clearly, the extension is ramified over the prime ideal $\mathfrak{p}=(2)$.

Remark 7. Table 1 above lists quadratic fields for some square-free discriminants $2 \leq D \leq 101$. The conductors $f$ and $\mathrm{f}$ satisfying equation (2) were calculated using tables for the class number of non-maximal orders in quadratic fields posted at www.numbertheory.org; the site is maintained by Keith Matthews. We focused on small conductors; the interested reader can compute the higher conductors using a pocket calculator. In contrast, computation of generator $x$ of the Hilbert class field require the online program $S A G E$ created by William A. Stein. We write an explicit formula for $x$ or its minimal polynomial $p(x)$ over $\mathfrak{k}$.

\section{Acknowledgment}

I thank Yu. I. Manin for helpful correspondence.

\section{References}

1. Hecke $E$ (1910) Concerning the construction of class field of real quadratic fields by means of automorphic functions, messages from the Gesselschaft of Sciences in Göttingen, Mathematics and Physical KI 6: 619-623.

2. Shimura G (1972) Class fields over real quadratic fields and Hecke operators Annals of Math 95: 130-190.

3. Stark HM (1976) $L$-functions at $s=1$. III. Totally Real Fields and Hilbert's Twelfth Problem. Advances in Math 22: 64-84.

4. Manin YI (2004) Real multiplication and noncommutative geometry. In: The legacy of Niels Hendrik Abel, Springer, Berlin pp: 685-727.

5. Silverman JH (1994) Advanced Topics in the Arithmetic of Elliptic Curves Graduate Texts in Mathematics, Springer.

6. Hecke $E$ (1928) Determination of the periods of certain integrals by the theory of class field. Math Z 28: 708-727.

7. Shimura G (1971) On elliptic curves with complex multiplication as factors of the Jacobians of modular function fields. Nagoya Math J 43: 199-208.

8. Stafford JT, van den Bergh M (2001) Noncommutative curves and noncommutative surfaces. Bull Amer Math Soc 38: 171-216.

9. Rieffel MA (1990) Non-commutative tori - A case study of non-commutative differentiable manifolds. Contemp Math 105: 191-211.

10. Borevich ZI, Shafarevich IR (1966) Number Theory, Acad Press, New York London.

11. Hasse H (1950) Lectures on Number Theory. Springer

12. Murphy GJ (1990) C*-Algebras and Operator Theory. Academic Press.

13. Blackadar B (1986) K-Theory for Operator Algebras. MSRI Publications, Springer.

14. Effros EG (1981) Dimensions and $C^{*}$-Algebras. In: Conf. Board of the Math Sciences, Amer Math Soc., Providence, RI.

15. Diamond F, Shurman J (2005) A First Course in Modular Forms. Number Theory and Discrete Mathematics, Springer.

16. Nikolaev I (2016) On a symmetry of complex and real multiplication. Hokkaido Math J 45: 43-51.

17. Bernstein L (1971) The Jacobi-Perron Algorithm, its Theory and Applications. Lect Notes in Math, Springer.

18. Nikolaev I (2012) On the AF-algebra of a Hecke eigenform. Proc Edinburgh Math Soc 55: 207-213

19. Cohen H, Roblot XF (2000) Computing the Hilbert class field of real quadratic fields. Math Comp 69: 1229-1244.
This article was originally published in a special issue, Recent Advances of Lie Theory in differential Geometry, in memory of John Nash handled by Editor. Dr. Princy Randriambololondrantomalala, Unversity of Antananarivo, Madagascar 